\title{
Optimal lower exponent for the higher gradient integrability of solutions to two-phase elliptic equations in two dimensions
}

\author{
Silvio Fanzon ${ }^{1} \cdot$ Mariapia Palombaro $^{1}$
}

Received: 23 March 2017 / Accepted: 30 July 2017 / Published online: 29 August 2017

(C) The Author(s) 2017. This article is an open access publication

\begin{abstract}
We study the higher gradient integrability of distributional solutions $u$ to the equation $\operatorname{div}(\sigma \nabla u)=0$ in dimension two, in the case when the essential range of $\sigma$ consists of only two elliptic matrices, i.e., $\sigma \in\left\{\sigma_{1}, \sigma_{2}\right\}$ a.e. in $\Omega$. In Nesi et al. (Ann Inst H Poincaré Anal Non Linéaire 31(3):615-638, 2014), for every pair of elliptic matrices $\sigma_{1}$ and $\sigma_{2}$, exponents $p_{\sigma_{1}, \sigma_{2}} \in(2,+\infty)$ and $q_{\sigma_{1}, \sigma_{2}} \in(1,2)$ have been found so that if $u \in W^{1, q_{\sigma_{1}, \sigma_{2}}(\Omega)}$ is solution to the elliptic equation then $\nabla u \in L_{\text {weak }}^{p_{\sigma_{1}, \sigma_{2}}}(\Omega)$ and the optimality of the upper exponent $p_{\sigma_{1}, \sigma_{2}}$ has been proved. In this paper we complement the above result by proving the optimality of the lower exponent $q_{\sigma_{1}, \sigma_{2}}$. Precisely, we show that for every arbitrarily small $\delta$, one can find a particular microgeometry, i.e., an arrangement of the sets $\sigma^{-1}\left(\sigma_{1}\right)$ and $\sigma^{-1}\left(\sigma_{2}\right)$, for which there exists a solution $u$ to the corresponding elliptic equation such that $\nabla u \in L^{q_{\sigma_{1}, \sigma_{2}}-\delta}$, but $\nabla u \notin L^{q_{\sigma_{1}, \sigma_{2}}}$. The existence of such optimal microgeometries is achieved by convex integration methods, adapting to the present setting the geometric constructions provided in Astala et al. (Ann Scuola Norm Sup Pisa Cl Sci 5(7):1-50, 2008) for the isotropic case.
\end{abstract}

Mathematics Subject Classification $30 \mathrm{C} 62 \cdot 35 \mathrm{~B} 27$

\section{Contents}

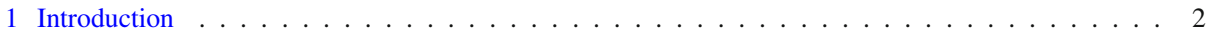

2 Connection with the Beltrami equation and explicit formulas for the optimal exponents . . . . . . . 3

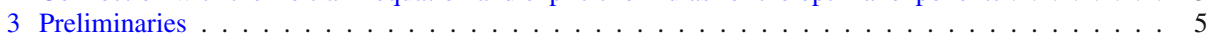

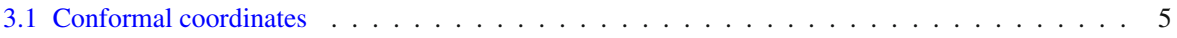

Communicated by C. De Lellis.

Mariapia Palombaro

M.Palombaro@sussex.ac.uk

Silvio Fanzon

S.Fanzon@sussex.ac.uk

1 Department of Mathematics, University of Sussex, Pevensey 2 Building, Falmer Campus,

Brighton BN1 9QH, UK 


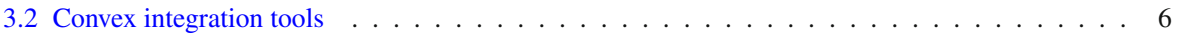

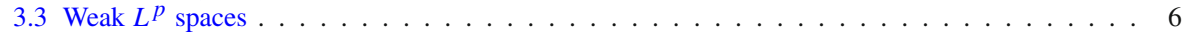

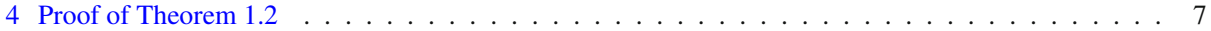

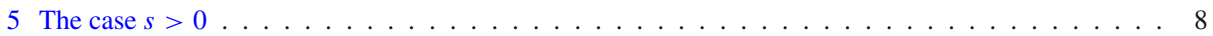

5.1 Properties of rank-one lines . . . . . . . . . . . . . . . . . . . . . . . 9

5.2 Weak staircase laminate . . . . . . . . . . . . . . . . . . . . . . . . . 14

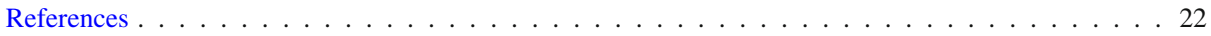

\section{Introduction}

Let $\Omega \subset \mathbb{R}^{2}$ be a bounded open domain and let $\sigma \in L^{\infty}\left(\Omega ; \mathbb{R}^{2 \times 2}\right)$ be uniformly elliptic, i.e.,

$$
\sigma \xi \cdot \xi \geq \lambda|\xi|^{2} \text { for every } \xi \in \mathbb{R}^{2} \text { and for a.e. } x \in \Omega,
$$

for some $\lambda>0$. We study the gradient integrability of distributional solutions $u \in W^{1,1}(\Omega)$ to

$$
\operatorname{div}(\sigma(x) \nabla u(x))=0 \quad \text { in } \Omega,
$$

in the case when the essential range of $\sigma$ consists of only two matrices, say $\sigma_{1}$ and $\sigma_{2}$. It is well-known from Astala's work [1] that there exist exponents $q$ and $p$, with $1<q<2<p$, such that if $u \in W^{1, q}(\Omega ; \mathbb{R})$ is solution to $(1.1)$, then $\nabla u \in L_{\text {weak }}^{p}(\Omega ; \mathbb{R})$. In [9] the optimal exponents $p$ and $q$ have been characterised for every pair of elliptic matrices $\sigma_{1}$ and $\sigma_{2}$. Denoting by $p_{\sigma_{1}, \sigma_{2}}$ and $q_{\sigma_{1}, \sigma_{2}}$ such exponents, whose precise formulas are recalled in Sect. 2, we summarise the result of [9] in the following theorem.

Theorem 1.1 [9, Theorem 1.4 and Proposition 4.2] Let $\sigma_{1}, \sigma_{2} \in \mathbb{R}^{2 \times 2}$ be elliptic.

(i) If $\sigma \in L^{\infty}\left(\Omega ;\left\{\sigma_{1}, \sigma_{2}\right\}\right)$ and $u \in W^{1, q_{\sigma_{1}, \sigma_{2}}}(\Omega)$ solves $(1.1)$, then $\nabla u \in L_{\text {weak }}^{p_{\sigma_{1}, \sigma_{2}}}\left(\Omega ; \mathbb{R}^{2}\right)$.

(ii) There exists $\bar{\sigma} \in L^{\infty}\left(\Omega ;\left\{\sigma_{1}, \sigma_{2}\right\}\right)$ and a weak solution $\bar{u} \in W^{1,2}(\Omega)$ to $(1.1)$ with $\sigma=\bar{\sigma}$, satisfying affine boundary conditions and such that $\nabla \bar{u} \notin L^{p_{\sigma_{1}, \sigma_{2}}}\left(\Omega ; \mathbb{R}^{2}\right)$.

Theorem 1.1 proves the optimality of the upper exponent $p_{\sigma_{1}, \sigma_{2}}$. The objective of this paper is to complement this result by proving the optimality of the lower exponent $q_{\sigma_{1}, \sigma_{2}}$. As shown in [9] (and recalled in Sect. 2), there is no loss of generality in assuming that

$$
\sigma_{1}=\operatorname{diag}\left(1 / K, 1 / S_{1}\right), \quad \sigma_{2}=\operatorname{diag}\left(K, S_{2}\right),
$$

with

$$
K>1 \quad \text { and } \quad \frac{1}{K} \leq S_{j} \leq K, \quad j=1,2 .
$$

Thus it suffices to show optimality for this class of coefficients, for which the exponents $p_{\sigma_{1}, \sigma_{2}}$ and $q_{\sigma_{1}, \sigma_{2}}$ read as

$$
q_{\sigma_{1}, \sigma_{2}}=\frac{2 K}{K+1}, \quad p_{\sigma_{1}, \sigma_{2}}=\frac{2 K}{K-1} .
$$

Our main result is the following

Theorem 1.2 Let $\sigma_{1}, \sigma_{2}$ be defined by (1.2) for some $K>1$ and $S_{1}, S_{2} \in[1 / K, K]$. There exist coefficients $\sigma_{n} \in L^{\infty}\left(\Omega,\left\{\sigma_{1} ; \sigma_{2}\right\}\right)$, exponents $p_{n} \in\left[1, \frac{2 K}{K+1}\right]$, functions $u_{n} \in$ $W^{1,1}(\Omega ; \mathbb{R})$ such that 


$$
\begin{aligned}
& \begin{cases}\operatorname{div}\left(\sigma_{n}(x) \nabla u_{n}(x)\right)=0 & \text { in } \Omega, \\
u_{n}(x)=x_{1} & \text { on } 2 \Omega,\end{cases} \\
& \nabla u_{n} \in L_{\text {weak }}^{p_{n}}\left(\Omega ; \mathbb{R}^{2}\right), \quad p_{n} \rightarrow \frac{2 K}{K+1}, \\
& \nabla u_{n} \notin L^{\frac{2 K}{K+1}}\left(\Omega ; \mathbb{R}^{2}\right) .
\end{aligned}
$$

In particular $u_{n} \in W^{1, q}(\Omega ; \mathbb{R})$ for every $q<p_{n}$, but $\int_{\Omega}\left|\nabla u_{n}\right|^{\frac{2 K}{K+1}} d x=\infty$.

Theorem 1.2 was proved in [2] in the case of isotropic coefficients, namely for $\sigma_{1}=$ $\frac{1}{K} I$ and $\sigma_{2}=K I$. More precisely, in [2] the authors obtain a slightly stronger result by constructing a single coefficient $\sigma \in\left\{K I, \frac{1}{K} I\right\}$ and a single function $u$ that satisfies the associated elliptic equation and is such that $\nabla u \in L_{\text {weak }}^{\frac{2 K}{K+1}}$, but $\nabla u \notin L^{\frac{2 K}{K+1}}$. We follow the method developed in [2], which relies on convex integration as used in [8], and provides an explicit construction of the sequence $u_{n}$. The adaptation of such method to the present context turns out to be non-trivial due to the anisotropy of the coefficients (see Remark 5.8). It is not clear how to modify the construction in order to get a stronger result as in [2].

\section{Connection with the Beltrami equation and explicit formulas for the optimal exponents}

For the reader's convenience we recall in this section how to reduce to the case (1.2) starting from any pair $\sigma_{1}, \sigma_{2}$. We will also give the explicit formulas for $p_{\sigma_{1}, \sigma_{2}}$ and $q_{\sigma_{1}, \sigma_{2}}$.

It is well-known that a solution $u \in W_{l o c}^{1, q}, q \geq 1$, to the elliptic equation (1.1) can be regarded as the real part of a complex map $f: \Omega \mapsto \mathbb{C}$ which is a $W_{l o c}^{1, q}$ solution to a Beltrami equation. Precisely, if $v$ is such that

$$
R_{\frac{\pi}{2}}^{T} \nabla v=\sigma \nabla u, \quad R_{\frac{\pi}{2}}:=\left(\begin{array}{cc}
0 & -1 \\
1 & 0
\end{array}\right),
$$

then $f:=u+i v$ solves the equation

$$
f_{\bar{z}}=\mu f_{z}+v \overline{f_{z}} \text { a.e. in } \Omega,
$$

where the so called complex dilatations $\mu$ and $\nu$, both belonging to $L^{\infty}(\Omega ; \mathbb{C})$, are given by

$$
\mu=\frac{\sigma_{22}-\sigma_{11}-i\left(\sigma_{12}+\sigma_{21}\right)}{1+\operatorname{Tr} \sigma+\operatorname{det} \sigma}, \quad v=\frac{1-\operatorname{det} \sigma+i\left(\sigma_{12}-\sigma_{21}\right)}{1+\operatorname{Tr} \sigma+\operatorname{det} \sigma},
$$

and satisfy the ellipticity condition

$$
\||\mu|+|\nu|\|_{L^{\infty}}<1 .
$$

The ellipticity (2.4) is often expressed in a different form. Indeed, it implies that there exists $0 \leq k<1$ such that $\||\mu|+|\nu|\|_{L^{\infty}} \leq k<1$ or equivalently that

$$
\||\mu|+|v|\|_{L^{\infty}} \leq \frac{K-1}{K+1},
$$

for some $K>1$. Let us recall that weak solutions to (2.2), (2.5) are called $K$-quasiregular mappings. Furthermore, we can express $\sigma$ as a function of $\mu, v$ inverting the algebraic system (2.3), 


$$
\sigma=\left(\begin{array}{cc}
\frac{|1-\mu|^{2}-|\nu|^{2}}{|1+\nu|^{2}-|\mu|^{2}} & \frac{2 \Im(\nu-\mu)}{|1+\nu|^{2}-|\mu|^{2}} \\
\frac{-2 \Im(\nu+\mu)}{|1+\nu|^{2}-|\mu|^{2}} & \frac{|1+\mu|^{2}-|\nu|^{2}}{|1+\nu|^{2}-|\mu|^{2}}
\end{array}\right)
$$

Conversely, if $f$ solves (2.2) with $\mu, v \in L^{\infty}(\Omega, \mathbb{C})$ satisfying (2.4), then its real part is solution to the elliptic equation (1.1) with $\sigma$ defined by (2.6). Notice that $\nabla f$ and $\nabla u$ enjoy the same integrability properties. Assume now that $\sigma: \Omega \rightarrow\left\{\sigma_{1}, \sigma_{2}\right\}$ is a two-phase elliptic coefficient and $f$ is solution to (2.2)-(2.3). Abusing notation, we identify $\Omega$ with a subset of $\mathbb{R}^{2}$ and $f=u+i v$ with the real mapping $f=(u, v): \Omega \rightarrow \mathbb{R}^{2}$. Then, as shown in [9], one can find matrices $A, B \in S L$ (2) (with $S L$ (2) denoting the set of invertible matrices with determinant equal to one) depending only on $\sigma_{1}$ and $\sigma_{2}$, such that, setting

$$
\tilde{f}(x):=A^{-1} f(B x),
$$

one has that the function $\tilde{f}$ solves the new Beltrami equation

$$
\tilde{f}_{\bar{z}}=\tilde{\mu} f_{z}+\tilde{v} \overline{\tilde{f}_{z}} \quad \text { a.e. in } B^{-1}(\Omega)
$$

and the corresponding $\tilde{\sigma}: B(\Omega) \rightarrow\left\{\tilde{\sigma}_{1}, \tilde{\sigma}_{2}\right\}$ defined by (2.6) is of the form (1.2):

$$
\tilde{\sigma}_{1}=\operatorname{diag}\left(1 / K, 1 / S_{1}\right), \quad \tilde{\sigma}_{2}=\operatorname{diag}\left(K, S_{2}\right), \quad K>1, \quad S_{1}, S_{2} \in[1 / K, K] .
$$

The results in $[1,12]$ imply that if $\tilde{f} \in W^{1, q}$, with $q \geq \frac{2 K}{K+1}$, then $\nabla \tilde{f} \in L_{\text {weak }}^{\frac{2 K}{K-1}}$; in particular, $\tilde{f} \in W^{1, p}$ for each $p<\frac{2 K}{K-1}$. Clearly $\nabla \tilde{f}$ enjoys the same integrability properties as $\nabla f$ and $\nabla u$.

Finally, we recall the formula for $K$ which will yield the optimal exponents. Denote by $d_{1}$ and $d_{2}$ the determinant of the symmetric part of $\sigma_{1}$ and $\sigma_{2}$ respectively,

$$
d_{i}:=\operatorname{det}\left(\frac{\sigma_{i}+\sigma_{i}^{T}}{2}\right), \quad i=1,2,
$$

and by $\left(\sigma_{i}\right)_{j k}$ the $j k$-entry of $\sigma_{i}$. Set

$$
\begin{aligned}
& m:=\frac{1}{\sqrt{d_{1} d_{2}}}\left[\left(\sigma_{2}\right)_{11}\left(\sigma_{1}\right)_{22}+\left(\sigma_{1}\right)_{11}\left(\sigma_{2}\right)_{22}-\frac{1}{2}\left(\left(\sigma_{2}\right)_{12}+\left(\sigma_{2}\right)_{21}\right)\left(\left(\sigma_{1}\right)_{12}+\left(\sigma_{1}\right)_{21}\right)\right] \\
& n:=\frac{1}{\sqrt{d_{1} d_{2}}}\left[\operatorname{det} \sigma_{1}+\operatorname{det} \sigma_{2}-\frac{1}{2}\left(\left(\sigma_{1}\right)_{21}-\left(\sigma_{1}\right)_{12}\right)\left(\left(\sigma_{2}\right)_{21}-\left(\sigma_{2}\right)_{12}\right)\right] .
\end{aligned}
$$

Then

$$
K=\left(\frac{m+\sqrt{m^{2}-4}}{2}\right)^{\frac{1}{2}}\left(\frac{n+\sqrt{n^{2}-4}}{2}\right)^{\frac{1}{2}}
$$

Thus, for any pair of elliptic matrices $\sigma_{1}, \sigma_{2} \in \mathbb{R}^{2 \times 2}$, the explicit formula for the optimal exponents $p_{\sigma_{1}, \sigma_{2}}$ and $q_{\sigma_{1}, \sigma_{2}}$ are obtained by plugging (2.8) into (1.4). 


\section{Preliminaries}

\subsection{Conformal coordinates}

For every real matrix $A \in \mathbb{R}^{2 \times 2}$,

$$
A=\left(\begin{array}{ll}
a_{11} & a_{12} \\
a_{21} & a_{22}
\end{array}\right)
$$

we write $A=\left(a_{+}, a_{-}\right)$, where $a_{+}, a_{-} \in \mathbb{C}$ denote its conformal coordinates. By identifying any vector $v=(x, y) \in \mathbb{R}^{2}$ with the complex number $v=x+i y$, conformal coordinates are defined by the identity

$$
A v=a_{+} v+a_{-} \bar{v} .
$$

Here $\bar{v}$ denotes the complex conjugation. From (3.1) we have relations

$$
a_{+}=\frac{a_{11}+a_{22}}{2}+i \frac{a_{21}-a_{12}}{2}, \quad a_{-}=\frac{a_{11}-a_{22}}{2}+i \frac{a_{21}+a_{12}}{2},
$$

and, conversely,

$$
\begin{array}{rlrl}
a_{11}=\Re a_{+}+\Re a_{-}, & & a_{12}=-\Im a_{+}+\Im a_{-}, \\
a_{21}=\Im a_{+}+\Im a_{-}, & a_{22}=\Re a_{+}-\Re a_{-} .
\end{array}
$$

Here $\mathfrak{R} z$ and $\mathfrak{\Im} z$ denote the real and imaginary part of $z \in \mathbb{C}$ respectively. We recall that

$$
A B=\left(a_{+} b_{+}+a_{-} \bar{b}_{-}, a_{+} b_{-}+a_{-} \bar{b}_{+}\right),
$$

and $\operatorname{Tr} A=2 \Re a_{+}$. Moreover

$$
\begin{aligned}
\operatorname{det}(A) & =\left|a_{+}\right|^{2}-\left|a_{-}\right|^{2}, \\
|A|^{2} & =2\left|a_{+}\right|^{2}+2\left|a_{-}\right|^{2}, \\
\|A\| & =\left|a_{+}\right|+\left|a_{-}\right|,
\end{aligned}
$$

where $|A|$ and $\|A\|$ denote the Hilbert-Schmidt and the operator norm, respectively.

We also define the second complex dilatation of the map $A$ as

$$
\mu_{A}:=\frac{a_{-}}{\bar{a}_{+}}
$$

and the distortion

$$
K(A):=\left|\frac{1+\left|\mu_{A}\right|}{1-\left|\mu_{A}\right|}\right|=\frac{\|A\|^{2}}{|\operatorname{det}(A)|} .
$$

The last two quantities measure how far $A$ is from being conformal. Following the notation introduced in [2], we define

$$
E_{\Delta}:=\{A=(a, \mu \bar{a}): a \in \mathbb{C}, \mu \in \Delta\}
$$

for a set $\Delta \subset \mathbb{C} \cup\{\infty\}$; namely, $E_{\Delta}$ is the set of matrices with the second complex dilatation belonging to $\Delta$. In particular $E_{0}$ and $E_{\infty}$ denote the set of conformal and anti-conformal matrices respectively. From (3.4) we have that $E_{\Delta}$ is invariant under precomposition by conformal matrices, that is

$$
E_{\Delta}=E_{\Delta} A \quad \text { for every } \quad A \in E_{0} \backslash\{0\} .
$$




\subsection{Convex integration tools}

We denote by $\mathcal{M}\left(\mathbb{R}^{2 \times 2}\right)$ the set of signed Radon measures on $\mathbb{R}^{2 \times 2}$ having finite mass. By the Riesz's representation theorem we can identify $\mathcal{M}\left(\mathbb{R}^{2 \times 2}\right)$ with the dual of the space $C_{0}\left(\mathbb{R}^{m \times n}\right)$. Given $v \in \mathcal{M}\left(\mathbb{R}^{2 \times 2}\right)$ we define its barycenter as

$$
\bar{v}:=\int_{\mathbb{R}^{2 \times 2}} A d v(A) .
$$

We say that a map $f \in C\left(\bar{\Omega} ; \mathbb{R}^{2}\right)$ is piecewise affine if there exists a countable family of pairwise disjoint open subsets $\Omega_{i} \subset \Omega$ with $\left|\partial \Omega_{i}\right|=0$ and

$$
\left|\Omega \backslash \bigcup_{i=1}^{\infty} \Omega_{i}\right|=0,
$$

such that $f$ is affine on each $\Omega_{i}$. Two matrices $A, B \in \mathbb{R}^{2 \times 2}$ such that $\operatorname{rank}(B-A)=1$ are said to be rank-one connected and the measure $\lambda \delta_{A}+(1-\lambda) \delta_{B} \in \mathcal{M}\left(\mathbb{R}^{2 \times 2}\right)$ with $\lambda \in[0,1]$ is called a laminate of first order (see also $[7,8,11]$ ).

Definition 3.1 The family of laminates of finite order $\mathcal{L}\left(\mathbb{R}^{2 \times 2}\right)$ is the smallest family of probability measures in $\mathcal{M}\left(\mathbb{R}^{2 \times 2}\right)$ satisfying the following conditions:

(i) $\delta_{A} \in \mathcal{L}\left(\mathbb{R}^{2 \times 2}\right)$ for every $A \in \mathbb{R}^{2 \times 2}$;

(ii) assume that $\sum_{i=1}^{N} \lambda_{i} \delta_{A_{i}} \in \mathcal{L}\left(\mathbb{R}^{2 \times 2}\right)$ and $A_{1}=\lambda B+(1-\lambda) C$ with $\lambda \in[0,1]$ and $\operatorname{rank}(B-C)=1$. Then the probability measure

$$
\lambda_{1}\left(\lambda \delta_{B}+(1-\lambda) \delta_{C}\right)+\sum_{i=2}^{N} \lambda_{i} \delta_{A_{i}}
$$

is also contained in $\mathcal{L}\left(\mathbb{R}^{2 \times 2}\right)$.

The process of obtaining new measures via (ii) is called splitting. The following proposition provides a fundamental tool to solve differential inclusions by means of convex integration (see e.g., [2, Proposition 2.3] or [8, Lemma 3.2] for a proof).

Proposition 3.2 Let $v=\sum_{i=1}^{N} \alpha_{i} \delta_{A_{i}} \in \mathcal{L}\left(\mathbb{R}^{2 \times 2}\right)$ be a laminate of finite order with barycenter $\bar{v}=A$, that is $A=\sum_{i=1}^{N} \alpha_{i} A_{i}$ with $\sum_{i=1}^{N} \alpha_{i}=1$. Let $\Omega \subset \mathbb{R}^{2}$ be a bounded open set, $\alpha \in(0,1)$ and $0<\delta<\min \left|A_{i}-A_{j}\right| / 2$. Then there exists a piecewise affine Lipschitz map $f: \Omega \rightarrow \mathbb{R}^{2}$ such that

(i) $f(x)=A x$ on $\partial \Omega$,

(ii) $[f-A]_{C^{\alpha}(\bar{\Omega})}<\delta$,

(iii) $\left|\left\{x \in \Omega:\left|\nabla f(x)-A_{i}\right|<\delta\right\}\right|=\alpha_{i}|\Omega|$,

(iv) $\operatorname{dist}(\nabla f(x), \operatorname{spt} v)<\delta$ a.e. in $\Omega$.

\subsection{Weak $L^{p}$ spaces}

We recall the definition of weak $L^{p}$ spaces. Let $f: \Omega \rightarrow \mathbb{R}^{2}$ be a Lebesgue measurable function. Define the distribution function of $f$ as

$$
\lambda_{f}:(0, \infty) \rightarrow[0, \infty] \text { with } \lambda_{f}(t):=|\{x \in \Omega:|f(x)|>t\}| .
$$


Let $1 \leq p<\infty$, then the following formula holds

$$
\int_{\Omega}|f(x)|^{p} d x=p \int_{0}^{\infty} t^{p-1} \lambda_{f}(t) d t .
$$

Define the quantity

$$
[f]_{p}:=\left(\sup _{t>0} t^{p} \lambda_{f}(t)\right)^{1 / p}
$$

and the weak $L^{p}$ space as

$$
L_{\text {weak }}^{p}\left(\Omega ; \mathbb{R}^{2}\right):=\left\{f: \Omega \rightarrow \mathbb{R}^{2}: f \text { measurable, }[f]_{p}<\infty\right\} .
$$

$L_{\text {weak }}^{p}$ is a topological vector space and by Chebyshev's inequality we have $[f]_{p} \leq\|f\|_{L^{p}}$. In particular this implies $L^{p} \subset L_{\text {weak }}^{p}$. Moreover $L_{\text {weak }}^{p} \subset L^{q}$ for every $q<p$.

\section{Proof of Theorem 1.2}

For the rest of this paper, $\sigma_{1}$ and $\sigma_{2}$ are as in (1.2)-(1.3). We start by rewriting (1.1) as a differential inclusion. To this end, define the sets

$$
T_{1}:=\left\{\left(\begin{array}{cc}
x & -y \\
S_{1}^{-1} y & K^{-1} x
\end{array}\right): x, y \in \mathbb{R}\right\}, \quad T_{2}:=\left\{\left(\begin{array}{cc}
x & -y \\
S_{2} y & K x
\end{array}\right): x, y \in \mathbb{R}\right\} .
$$

Let $\sigma \in L^{\infty}\left(\Omega ;\left\{\sigma_{1}, \sigma_{2}\right\}\right)$. It is easy to check (see for example [2, Lemma 3.2]) that $u$ solves (1.1) if and only if $f$ solves the differential inclusion

$$
\nabla f(x) \in T_{1} \cup T_{2} \text { a.e. in } \Omega,
$$

where $f:=(u, v)$ and $v$ is the stream function of $u$, which is defined, up to an addictive constant, by (2.1).

In order to solve the differential inclusion (4.2), it is convenient to use (3.2) and write our target sets in conformal coordinates:

$$
T_{1}=\left\{\left(a, d_{1}(\bar{a})\right): a \in \mathbb{C}\right\}, \quad T_{2}=\left\{\left(a,-d_{2}(\bar{a})\right): a \in \mathbb{C}\right\},
$$

where the operators $d_{j}: \mathbb{C} \rightarrow \mathbb{C}$ are defined as

$$
d_{j}(a):=k \Re a+i s_{j} \Im a, \text { with } k:=\frac{K-1}{K+1} \quad \text { and } \quad s_{j}:=\frac{S_{j}-1}{S_{j}+1} .
$$

Conditions (1.3) imply

$$
0<k<1 \text { and }-k \leq s_{j} \leq k \text { for } j=1,2 .
$$

Introduce the quantities

$$
\begin{array}{r}
s:=\frac{s_{1}+s_{2}}{2}=\frac{S_{1} S_{2}-1}{\left(1+S_{1}\right)\left(1+S_{2}\right)} \\
S:=\frac{1+s}{1-s}=\frac{S_{1}+S_{2}+2 S_{1} S_{2}}{2+S_{1}+S_{2}} .
\end{array}
$$


By (4.5) we have

$$
-k \leq s \leq k \quad \text { and } \quad \frac{1}{K} \leq S \leq K .
$$

We distinguish three cases.

1. Case $s>0$ (corresponding to $S>1$ ). We study this case in Sect. 5, where we generalise the methods used in [2, Section 3.2]. Observe that this case includes the one studied in [2]. Indeed, for $s=k$ one has that $s_{1}=s_{2}=k$ and the target sets (4.3) become

$$
T_{1}=E_{k}=\{(a, k \bar{a}): a \in \mathbb{C}\}, \quad T_{2}=E_{-k}=\{(a,-k \bar{a}): a \in \mathbb{C}\},
$$

where $E_{ \pm k}$ are defined in (3.8). We remark that, in this particular case, the construction provided in Section 5 coincides with the one given in [2, Section 3.2].

2. Case $s<0$ (corresponding to $S<1$ ). This case can be reduced to the previous one. Indeed, if we introduce $\hat{s}_{j}:=-s_{j}, \hat{s}:=\left(\hat{s}_{1}+\hat{s}_{2}\right) / 2>0$ and the operators $\hat{d}_{j}(a):=k \Re a+i \hat{s}_{j} \Im a$ then the target sets (4.3) read as

$$
T_{1}=\left\{\left(a, \hat{d}_{1}(a)\right): a \in \mathbb{C}\right\}, \quad T_{2}=\left\{\left(a,-\hat{d}_{2}(a)\right): a \in \mathbb{C}\right\} .
$$

This is the same as the previous case, since the absence of the conjugation does not affect the geometric properties relevant to the constructions of Sect. 5.

We notice that this case includes $s=-k$ for which the target sets become

$$
T_{1}=\{(a, k a): a \in \mathbb{C}\}, \quad T_{2}=\{(a,-k a): a \in \mathbb{C}\} .
$$

We remark that in this case, (4.2) coincides with the classical Beltrami equation (see also [2, Remark 3.21]).

3. Case $s=0$ (corresponding to $s_{1}=-s_{2}, S_{1}=1 / S_{2}$ ) This is a degenerate case, in the sense that the constructions provided in Section 5 for $s>0$ are not well defined. Nonetheless, Theorem 1.2 still holds true. In fact, as already pointed out in [9, Section A.3], by an affine change of variables, the existence of a solution can be deduced by [2, Lemma 4.1, Theorem 4.14], where the authors prove the optimality of the lower critical exponent $\frac{2 K}{K+1}$ for the solution of a system in non-divergence form. We remark that in this case Theorem 1.2 actually holds in the stronger sense of exact solutions, namely, there exists $u \in W^{1,1}(\Omega ; \mathbb{R})$ solution to (1.5) and such that

$$
\nabla u \in L_{\text {weak }}^{\frac{2 K}{K+1}}\left(\Omega ; \mathbb{R}^{2}\right), \quad \nabla u \notin L^{\frac{2 K}{K+1}}\left(\Omega ; \mathbb{R}^{2}\right) .
$$

\section{The case $s>0$}

In the present section we prove Theorem 1.2 under the hypothesis that the average $s$ is positive, namely that

$$
\begin{aligned}
& 0<k<1 \text { and }-s_{2}<s_{1} \leq s_{2}, \\
& 0<k<1 \text { with } 0<s_{2} \leq k, \text { or } \\
& -s_{1}<s_{2} \leq s_{1},
\end{aligned}
$$

From (5.1), recalling definitions (4.4), (4.6), (4.7), we have

$$
\begin{aligned}
& 0<s \leq k, \quad 1<S \leq K, \\
& 1 / S_{2}<S_{1} \leq S_{2}, \quad 1<S_{2} \leq K, \quad \text { or } \quad 1 / S_{1}<S_{2} \leq S_{1}, \quad 1<S_{1} \leq K
\end{aligned}
$$

In order to prove Theorem 1.2, we will solve the differential inclusion (4.2) by adapting the convex integration program developed in [2, Section 3.2] to the present context. As already 
pointed out in the Introduction, the anisotropy of the coefficients $\sigma_{1}, \sigma_{2}$ poses some technical difficulties in the construction of the so-called staircase laminate, needed to obtain the desired approximate solutions. In fact, the anisotropy of $\sigma_{1}, \sigma_{2}$ translates into the lack of conformal invariance (in the sense of (3.9)) of the target sets (4.3), while the constructions provided in [2] heavily rely on the conformal invariance of the target set $E_{\{-k, k\}}$. We point out that the lack of conformal invariance was a source of difficulty in [9] as well, for the proof of the optimality of the upper exponent.

This section is divided as follows. In Sect. 5.1 we establish some geometrical properties of rank-one lines in $\mathbb{R}^{2 \times 2}$, that will be used in Sect. 5.2 for the construction of the staircase laminate. For every sufficiently small $\delta>0$, such laminate allows us to define (in Proposition 5.9) a piecewise affine map $f$ that solves the differential inclusion (4.2) up to an arbitrarily small $L^{\infty}$ error. Moreover $f$ will have the desired integrability properties (see (5.59), that is,

$$
\nabla f \in L_{\text {weak }}^{p}\left(\Omega ; \mathbb{R}^{2 \times 2}\right), \quad p \in\left(\frac{2 K}{K+1}-\delta, \frac{2 K}{K+1}\right], \quad \nabla f \notin L^{\frac{2 K}{K+1}}\left(\Omega ; \mathbb{R}^{2 \times 2}\right) .
$$

Finally, in Theorem 5.10, we remove the $L^{\infty}$ error introduced in Proposition 5.9, by means of a standard argument (see, e.g., [9, Theorem A.2]).

Throughout this section $c_{K}>1$ will denote various constants depending on $K, S_{1}$ and $S_{2}$, whose precise value may change from place to place. The complex conjugation is denoted by $J:=(0,1)$ in conformal coordinates, i.e., $J z=\bar{z}$ for $z \in \mathbb{C}$. Moreover, $R_{\theta}:=\left(e^{i \theta}, 0\right) \in$ $S O$ (2) denotes the counter clockwise rotation of angle $\theta \in(-\pi, \pi]$. Define the the argument function

$$
\arg z:=\theta, \text { where } z=|z| e^{i \theta} \text {, with } \theta \in(-\pi, \pi] .
$$

Abusing notation we write $\arg R_{\theta}=\theta$. For $A=(a, b) \in \mathbb{R}^{2 \times 2} \backslash\{0\}$ we set

$$
\theta_{A}:=-\arg \left(b-d_{1}(\bar{a})\right) .
$$

\subsection{Properties of rank-one lines}

In this Section we will establish some geometrical properties of rank-one lines in $\mathbb{R}^{2 \times 2}$. Lemmas 5.2, 5.3 are generalizations of [2, Lemmas 3.14, 3.15] to our target sets (4.3). In Lemmas 5.4, 5.5 we will study certain rank-one lines connecting $T$ to $E_{\infty}$, that will be used in Sect. 5.2 to construct the staircase laminate.

Lemma 5.1 Let $Q \in T_{j}$ with $j \in\{1,2\}$ and $T_{j}$ as in (4.3). Then

$$
\begin{aligned}
& \text { det } Q>0 \quad \text { for } \quad Q \neq 0, \\
& \left|s_{j}\right| \leq\left|\mu_{Q}\right| \leq k, \\
& \max \left\{S_{j}, 1 / S_{j}\right\} \leq K(Q) \leq K .
\end{aligned}
$$

Proof Let $Q=\left(q, d_{1}(\bar{q})\right) \in T_{1}$. By (4.5) we have $\left|s_{1}\right||q| \leq\left|d_{1}(q)\right| \leq k|q|$ which readily implies (5.6) and

$$
\left(1-k^{2}\right)|q|^{2} \leq \operatorname{det}(Q) \leq\left(1-s_{1}^{2}\right)|q|^{2} .
$$

The last inequality implies (5.5). Finally $K(Q)$ is increasing with respect to $\left|\mu_{Q}\right| \in(0,1)$, therefore (5.7) follows from (5.6). The proof is analogous if $Q \in T_{2}$. 
Lemma 5.2 Let $A, B \in \mathbb{R}^{2 \times 2}$ with $\operatorname{det} B \neq 0$ and $\operatorname{det}(B-A)=0$, then

$$
|B| \leq \sqrt{2} K(B)|A| .
$$

In particular, if $A \in \mathbb{R}^{2 \times 2}$ and $Q \in T_{j}, j \in\{1,2\}$, are such that $\operatorname{det}(A-Q)=0$, then

$$
\operatorname{dist}\left(A, T_{j}\right) \leq|A-Q| \leq(1+\sqrt{2} K) \operatorname{dist}\left(A, T_{j}\right) .
$$

Proof The first part of the statement is exactly like in [2, Lemma 3.14]. For the second part, one can easily adapt the proof of [2, Lemma 3.14] to the present context taking into account (5.5) and (5.7). For the reader's convenience we recall the argument. Let $A \in \mathbb{R}^{2 \times 2}, Q \in T_{1}$ and $Q_{0} \in T_{1}$ such that $\operatorname{dist}\left(A, T_{1}\right)=\left|A-Q_{0}\right|$. By (5.5), we can apply the first part of the lemma to $A-Q_{0}$ and $Q-Q_{0}$ to get

$$
\left|Q-Q_{0}\right| \leq \sqrt{2} K\left(Q-Q_{0}\right)\left|A-Q_{0}\right| \leq \sqrt{2} K\left|A-Q_{0}\right|,
$$

where the last inequality follows from (5.7), since $Q-Q_{0} \in T_{1}$. Therefore

$$
|A-Q| \leq\left|A-Q_{0}\right|+\left|Q-Q_{0}\right| \leq(1+\sqrt{2} K)\left|A-Q_{0}\right|=(1+\sqrt{2} K) \operatorname{dist}\left(A, T_{1}\right) .
$$

The proof for $T_{2}$ is analogous.

Lemma 5.3 Every $A=(a, b) \in \mathbb{R}^{2 \times 2} \backslash\{0\}$ lies on a rank-one segment connecting $T_{1}$ and $E_{\infty}$. Precisely, there exist matrices $Q \in T_{1} \backslash\{0\}$ and $P \in E_{\infty} \backslash\{0\}$, with $\operatorname{det}(P-Q)=0$, such that $A \in[Q, P]$. We have $P=t J R_{\theta_{A}}$ for some $t>0$ and $\theta_{A}$ as in (5.4). Moreover, there exists a constant $c_{K}>1$, depending only on $K, S_{1}, S_{2}$, such that

$$
\frac{1}{c_{K}}|A| \leq|P-Q|,|P|,|Q| \leq c_{K}|A| \text {. }
$$

Proof The proof can be deduced straightforwardly from the one of [2, Lemma 3.15]. We decompose any $A=(a, b)$ as

$$
A=\left(a, d_{1}(\bar{a})\right)+\frac{1}{t}\left(0, t b-t d_{1}(\bar{a})\right)=Q+\frac{1}{t} P_{t},
$$

with $Q \in T_{1}$ and $P_{t} \in E_{\infty}$. The matrices $Q$ and $P_{t}$ are rank-one connected if and only if $|a|=\left|d_{1}(\bar{a})+t\left(b-d_{1}(\bar{a})\right)\right|$. Since $\operatorname{det} Q>0$ for $Q \neq 0$, it is easy to see that there exists only one $t_{0}>0$ such that the last identity is satisfied. We then set $\rho:=1+1 / t_{0}$ so that

$$
A=\frac{1}{\rho}(\rho Q)+\frac{1}{t_{0} \rho}\left(\rho P_{t_{0}}\right) .
$$

The latter is the desired decomposition, since $\rho Q \in T_{1}, \rho P_{t_{0}} \in E_{\infty}$ are rank-one connected, $\rho>0$ and $\rho^{-1}+\left(t_{0} \rho\right)^{-1}=1$. Also notice that $\rho P_{t_{0}}=\rho t_{0}\left|b-d_{1}(\bar{a})\right| J R_{\theta_{A}}$ as stated.

Finally let us prove (5.9). Remark that

$$
\operatorname{dist}\left(A, T_{1}\right)+\operatorname{dist}\left(A, E_{\infty}\right) \leq|A-P|+|A-Q|=|P-Q| .
$$

By the linear independence of $T_{1}$ and $E_{\infty}$, we get

$$
\frac{1}{c_{K}}|A| \leq|P-Q| \text {. }
$$

Using Lemma 5.2, (5.5) and (5.7) we obtain

$$
|P| \leq c_{K}|A|, \quad|Q| \leq c_{K}|A|, \quad|Q| \leq c_{K}|P|, \quad|P| \leq c_{K}|Q| .
$$


By the triangle inequality,

$$
|P-Q| \leq|P|+|Q| \leq\left(1+c_{K}\right) \min (|P|,|Q|),
$$

and (5.9) follows.

We now turn our attention to the study of rank-one connections between the target set $T$ and $E_{\infty}$.

Lemma 5.4 Let $R=(r, 0)$ with $|r|=1$ and $a \in \mathbb{C} \backslash\{0\}$. For $j \in\{1,2\}$ define

$$
\begin{aligned}
& Q_{1}(a):=\lambda_{1}\left(a, d_{1}(\bar{a})\right) \in T_{1}, \quad Q_{2}(a):=\lambda_{2}\left(-a, d_{2}(\bar{a})\right) \in T_{2}, \\
& \lambda_{j}(a):=\frac{1}{\sqrt{B_{j}^{2}(a)+A_{j}(a)}+B_{j}(a)}, \\
& \left\{\begin{array}{l}
A_{j}(a):=\operatorname{det}\left(a, d_{j}(a)\right)=|a|^{2}-\left|d_{j}(a)\right|^{2}, \\
B_{j}(a):=\Re\left(\bar{r} d_{j}(a)\right) .
\end{array}\right.
\end{aligned}
$$

Then $\lambda_{j}>0, A_{j}>0$ and $\operatorname{det}\left(Q_{j}-J R\right)=0$. Moreover there exists a constant $c_{K}>1$ depending only on $K, S_{1}, S_{2}$ such that

$$
\frac{1}{c_{K}} \leq\left|Q_{j}(a)\right| \leq c_{K}
$$

for every $a \in \mathbb{C} \backslash\{0\}$ and $R \in S O(2)$.

Proof Condition $\operatorname{det}\left(Q_{j}-J R\right)=0$ is equivalent to $\left|\lambda_{j} a\right|=\left|\lambda_{j} d_{j}(\bar{a})-\bar{r}\right|$, that is

$$
A_{j}(a) \lambda_{j}^{2}+2 B_{j}(a) \lambda_{j}-1=0
$$

with $A_{j}, B_{j}$ defined by (5.11). Notice that $A_{j}>0$ by (5.5). Therefore $\lambda_{j}$ defined in (5.10) solves (5.13) and satisfies $\lambda_{j}>0$.

We will now prove (5.12). Since $a \neq 0$, we can write $a=t \omega$ for some $t>0$ and $\omega \in \mathbb{C}$, with $|\omega|=1$. We have $A_{j}(a)=t^{2} A_{j}(\omega)$ and $B_{j}(a)=t B_{j}(\omega)$ so that $\lambda_{j}(a)=\lambda_{j}(\omega) / t$. Hence

$$
Q_{1}(a)=\lambda_{1}(\omega)\left(\omega, d_{1}(\bar{\omega})\right), \quad Q_{2}(a)=\lambda_{2}(\omega)\left(-\omega, d_{2}(\bar{\omega})\right) .
$$

Since $\lambda_{j}$ is continuous and positive in $(\mathbb{C} \backslash\{0\}) \times S O(2),(5.12)$ follows from (5.14).

Notation. Let $\theta \in(-\pi, \pi]$. For $R_{\theta}=\left(e^{i \theta}, 0\right) \in S O(2)$, define $x:=\cos \theta, y:=\sin \theta$ and

$$
a\left(R_{\theta}\right):=\frac{x}{k}+i \frac{y}{s}
$$

where $s$ is defined in (4.6). Identifying $S O(2)$ with the interval $(-\pi, \pi]$, for $j=1,2$, we introduce the function

$$
\lambda_{j}:(-\pi, \pi] \rightarrow(0,+\infty) \text { defined by } \lambda_{j}\left(R_{\theta}\right):=\lambda_{j}\left(a\left(R_{\theta}\right)\right)
$$

with $\lambda_{j}\left(a\left(R_{\theta}\right)\right)$ as in (5.10). Furthermore, for $n \in \mathbb{N}$ set

$$
\begin{aligned}
M_{j}\left(R_{\theta}\right) & :=\frac{\lambda_{j}}{\frac{\lambda_{1}+\lambda_{2}}{2}-\lambda_{1} \lambda_{2}}, \quad l\left(R_{\theta}\right):=\frac{M_{1}+M_{2}}{2}-1, \quad m:=\min _{\theta \in(-\pi, \pi]} \frac{M_{2}}{2-M_{2}} \\
L\left(R_{\theta}\right) & :=\frac{1+l}{1-l}, \quad \beta_{n}\left(R_{\theta}\right):=1-\frac{1+l}{n}, \quad p\left(R_{\theta}\right):=\frac{2 L}{L+1} .
\end{aligned}
$$


Lemma 5.5 For $j=1,2$, the functions

$$
\begin{aligned}
& \lambda_{j}:(-\pi, \pi] \rightarrow\left[\frac{s}{1+s_{j}}, \frac{k}{1+k}\right], \quad l:(-\pi, \pi] \rightarrow[s, k], \\
& L:(-\pi, \pi] \rightarrow[S, K], \quad p:(-\pi, \pi] \rightarrow\left[\frac{2 S}{S+1}, \frac{2 K}{K+1}\right],
\end{aligned}
$$

are even, surjective and their periodic extension is $C^{1}$. Furthermore, they are strictly decreasing in $(0, \pi / 2)$ and strictly increasing in $(\pi / 2, \pi)$, with maximum at $\theta=0, \pi$ and minimum at $\theta=\pi / 2$. Finally

$$
\begin{aligned}
& 0<M_{j}<2, \quad m>0, \\
& \prod_{j=1}^{n} \beta_{j}\left(R_{\theta}\right)=\frac{1}{n^{p\left(R_{\theta}\right)}}+O\left(\frac{1}{n}\right),
\end{aligned}
$$

where $O(1 / n) \rightarrow 0$ as $n \rightarrow \infty$ uniformly for $\theta \in(-\pi, \pi]$.

Proof Let us consider $\lambda_{j}$ first. By definitions (5.11), (5.15) and by recalling that $x^{2}+y^{2}=1$, we may regard $A_{j}, B_{j}$ and $\lambda_{j}$ as functions of $x \in[-1,1]$. In particular,

$$
A_{j}(x)=\left(\frac{1-k^{2}}{k^{2}}-\frac{1-s_{j}^{2}}{s^{2}}\right) x^{2}+\frac{1-s_{j}^{2}}{s^{2}}, \quad B_{j}(x)=\left(1-\frac{s_{j}}{s}\right) x^{2}+\frac{s_{j}}{s} .
$$

By symmetry we can restrict to $x \in[0,1]$. We have three cases:

1. Case $s_{1}=s_{2}$. Since $s_{1}=s_{2}=s$, from (5.20) we compute

$$
\lambda_{1}(x)=\lambda_{2}(x)=\left(1+\sqrt{\left(\frac{1}{k^{2}}-\frac{1}{s^{2}}\right) x^{2}+\frac{1}{s^{2}}}\right)^{-1} .
$$

By (5.1),(5.2) this is a strictly increasing function in $[0,1]$, and the rest of the thesis for $\lambda_{j}$ readily follows.

2. Case $s_{1}<s_{2}$. By (5.1) we have

$$
-s_{2}<s_{1}<s \quad \text { and } \quad 0<s<s_{2} .
$$

Relations (5.20) and (5.21) imply that

$$
\begin{aligned}
& A_{j}^{\prime}(0)=0, \quad A_{j}^{\prime}(x)<0, \quad \text { for } \quad x \in(0,1], \\
& B_{1}^{\prime}(0)=0, \quad B_{1}^{\prime}(x)>0, \quad \text { for } \quad x \in(0,1] \text {, } \\
& B_{2}^{\prime}(0)=0, \quad B_{2}^{\prime}(x)<0, \quad \text { for } \quad x \in(0,1] \text {. }
\end{aligned}
$$

We claim that

$$
\lambda_{j}^{\prime}(0)=0, \quad \lambda_{j}^{\prime}(x)>0, \quad \text { for } \quad x \in(0,1]
$$

Before proving (5.25), notice that $\lambda_{j}(0)=\frac{s}{1+s_{j}}$ and $\lambda_{j}(1)=\frac{k}{1+k}$, therefore the surjectivity of $\lambda_{j}$ will follow from (5.25). Let us now prove (5.25). For $j=2$ condition (5.25) is an immediate consequence of the definition of $\lambda_{2}$ and (5.22), (5.24). For $j=1$ we have 


$$
\lambda_{1}^{\prime}(x)=-\frac{1}{\lambda_{1}^{2}}\left(\frac{A_{1}^{\prime}+2 B_{1} B_{1}^{\prime}}{2 \sqrt{B_{1}^{2}+A_{1}}}+B_{1}^{\prime}\right)
$$

and we immediately see that $\lambda_{1}^{\prime}(0)=0$ by (5.22) and (5.23). Assume now that $x \in(0,1]$. By (5.23) and (5.26), the claim (5.25) is equivalent to

$$
A_{1}^{\prime 2}+4 A_{1}^{\prime} B_{1} B_{1}^{\prime}-4 A_{1} B_{1}^{\prime 2}>0, \quad \text { for } \quad x \in(0,1] .
$$

After simplifications, the above inequality is equivalent to

$$
\frac{4 f\left(s_{1}, s_{2}\right)}{k^{4}\left(s_{1}+s_{2}\right)^{4}} x^{2}>0, \quad \text { for } \quad x \in(0,1],
$$

where $f\left(s_{1}, s_{2}\right)=a b c d$, with

$$
\begin{aligned}
a & =-2 k+(1+k) s_{1}+(1-k) s_{2}, & b & =2 k+(1+k) s_{1}+(1-k) s_{2}, \\
c & =-2 k-(1-k) s_{1}-(1+k) s_{2}, & d & =2 k-(1-k) s_{1}-(1+k) s_{2} .
\end{aligned}
$$

We have that $a, c<0$ since $s_{1}<s_{2}$ and $b, d>0$ since $s_{1}>-s_{2}$. Hence (5.27) follows.

3. Case $s_{2}<s_{1}$. In particular we have

$$
-s_{1}<s_{2}<s \quad \text { and } \quad 0<s<s_{1} .
$$

This is similar to the previous case. Indeed (5.22) is still true, but for $B_{j}$ we have

$$
\begin{aligned}
& B_{1}^{\prime}(0)=0, \quad B_{1}^{\prime}(x)<0, \quad \text { for } \quad x \in(0,1], \\
& B_{2}^{\prime}(0)=0, \quad B_{2}^{\prime}(x)>0, \quad \text { for } \quad x \in(0,1] .
\end{aligned}
$$

This implies (5.25) with $j=1$. Similarly to the previous case, we can see that (5.25) for $j=2$ is equivalent to

$$
\frac{4 f\left(s_{2}, s_{1}\right)}{k^{4}\left(s_{1}+s_{2}\right)^{4}} x^{2}>0, \quad \text { for } \quad x \in(0,1] .
$$

Notice that $f$ is symmetric, therefore (5.31) is a consequence of (5.27).

We will now turn our attention to the function $l$. Notice that

$$
l=\frac{1}{1-H}-1, \quad \text { where } H:=\frac{2 \lambda_{1} \lambda_{2}}{\lambda_{1}+\lambda_{2}}=2\left(\frac{1}{\lambda_{1}}+\frac{1}{\lambda_{2}}\right)^{-1}
$$

is the harmonic mean of $\lambda_{1}$ and $\lambda_{2}$. Therefore $H$ is differentiable and even. By direct computation we have

$$
H^{\prime}=2 \frac{\lambda_{1}^{\prime} \lambda_{2}^{2}+\lambda_{1}^{2} \lambda_{2}^{\prime}}{\left(\lambda_{1}+\lambda_{2}\right)^{2}}
$$

Since $\lambda_{j}>0$, by (5.25) we have

$$
H^{\prime}(0)=0, \quad H^{\prime}(x)>0, \quad \text { for } \quad x \in(0,1] .
$$

Moreover $H(0)=\frac{s}{1+s}$ and $H(1)=\frac{k}{1+k}$. Then from (5.32) we deduce $l(0)=s, l(1)=k$ and the rest of the statement for $l$.

The statements for $L$ and $p$ follow directly from the properties of $l$ and from the fact that $t \rightarrow \frac{1+t}{1-t}, t \rightarrow \frac{2 t}{t+1}$ are $C^{1}$ and strictly increasing for $0<t<1$ and $t>1$, respectively. 
Next we prove (5.18). By (5.1) and the properties of $\lambda_{j}$, we have in particular

$$
0<\lambda_{j}<\frac{1}{2}, \quad 0<H<\frac{1}{2},
$$

where $H$ is defined in (5.32). Since $\lambda_{j}>0$, the inequality $M_{j}>0$ is equivalent to $H<1$, which holds by (5.34). The inequality $M_{2}<2$ is instead equivalent to $\lambda_{1}\left(1-2 \lambda_{2}\right)>0$, which is again true by (5.34). The case $M_{1}<2$ is similar. Finally $m>0$ follows from $0<M_{2}<2$ and the continuity of $\lambda_{j}$.

Finally we prove (5.19). By definition we have $1+l=\frac{2 L}{L+1}=p$. By taking the logarithm of $\prod_{j=1}^{n} \beta_{j}\left(R_{\theta}\right)$, we see that there exists a constant $c>0$, depending only on $K, S_{1}, S_{2}$, such that

$$
\left|\log \left(\prod_{j=1}^{n} \beta_{j}\left(R_{\theta}\right)\right)+p\left(R_{\theta}\right) \log n\right|<c, \text { for every } \theta \in(-\pi, \pi] .
$$

Estimate (5.35) is uniform because $\beta_{j}$ and $p$ are $\pi$-periodic and uniformly continuous.

\subsection{Weak staircase laminate}

We are now ready to construct a staircase laminate in the same fashion as [2, Lemma 3.17]. We remark that the construction of this type of laminates, first introduced in [5], has also been used in $[3,4]$ in connection with the problem of regularity for rank-one convex functions and in $[6,10]$ for constructing Sobolev homeomorphisms with gradients of law rank.

The steps of our staircase will be the sets

$$
\mathcal{S}_{n}:=n J S O(2)=\left\{\left(0, n e^{i \theta}\right): \theta \in(-\pi, \pi]\right\}, \quad n \geq 1 .
$$

For $0<\delta<\pi / 2$ we introduce the set

$$
E_{\infty}^{\delta}:=\left\{(0, z) \in E_{\infty}:|\arg z|<\delta\right\}, \quad \mathcal{S}_{n}^{\delta}:=\mathcal{S}_{n} \cap E_{\infty}^{\delta} .
$$

Lemma 5.6 Let $0<\delta<\pi / 4$ and $0<\rho<\min \left\{m, \frac{1}{2}\right\}$, with $m>0$ defined in (5.17). There exists a constant $c_{K}>1$ depending only on $K, S_{1}, S_{2}$, such that for every $A=(a, b) \in \mathbb{R}^{2 \times 2}$ satisfying

$$
\operatorname{dist}\left(A, \mathcal{S}_{n}\right)<\rho,
$$

there exists a laminate of third order $v_{A}$, such that:

(i) $\bar{v}_{A}=A$,

(ii) $\operatorname{spt} v_{A} \subset T \cup \mathcal{S}_{n+1}$,

(iii) $\operatorname{spt} v_{A} \subset\left\{\xi \in \mathbb{R}^{2 \times 2}: c_{K}^{-1} n<|\xi|<c_{K} n\right\}$,

(iv) $\operatorname{spt} v_{A} \cap \mathcal{S}_{n+1}=\{(n+1) J R\}$, with $R=R_{\theta_{A}}$ as in (5.4).

Moreover

$$
\left(1-c_{K} \frac{\rho}{n}\right) \beta_{n}(R) \leq v_{A}\left(\mathcal{S}_{n+1}\right) \leq\left(1+c_{K} \frac{\rho}{n}\right) \beta_{n+2}(R),
$$

where $\beta_{n}$ is defined in (5.17). If in addition $n \geq 2$ and

$$
\operatorname{dist}\left(A, \mathcal{S}_{n}^{\delta}\right)<\rho,
$$


Fig. 1 Weak staircase laminate

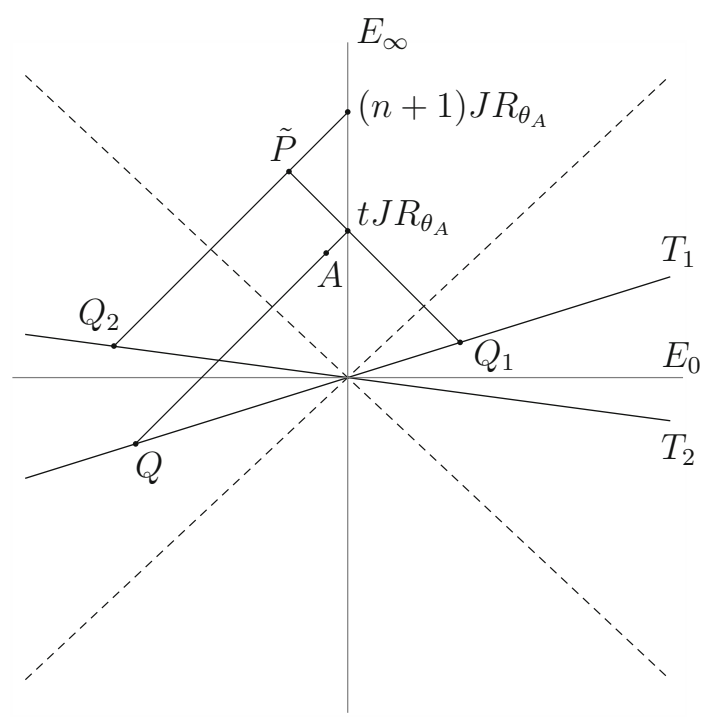

then

$$
|\arg R|=\left|\theta_{A}\right|<\delta+\rho .
$$

In particular spt $v_{A} \subset T \cup \mathcal{S}_{n+1}^{\delta+\rho}$.

Proof Let us start by defining $v_{A}$. From Lemma 5.3 there exist $c_{K}>1$ and non zero matrices $Q \in T_{1}, P \in E_{\infty}$, such that $\operatorname{det}(P-Q)=0$,

$$
\begin{aligned}
& A=\mu_{1} Q+\left(1-\mu_{1}\right) P, \quad \text { for some } \mu_{1} \in[0,1], \\
& \frac{1}{c_{K}}|A| \leq|P-Q|,|P|,|Q| \leq c_{K}|A| .
\end{aligned}
$$

Moreover $P=t J R$ with $R=R_{\theta_{A}}=(r, 0)$ as in (5.4) and $t>0$. We will estimate $t$. By (5.36), there exists $\tilde{R} \in S O$ (2) such that $|A-n J \tilde{R}|<\rho$. Applying Lemma 5.2 to $A-n J \tilde{R}$ and $P-n J \tilde{R}$ yields

$$
|P-n J \tilde{R}|<\sqrt{2} \rho,
$$

since $P-n J \tilde{R} \in E_{\infty}$. Hence from (5.42) we get

$$
|t-n|<\rho,
$$

since $|J R|=|J \tilde{R}|=\sqrt{2}$. We also have

$$
\mu_{1}=\frac{|A-Q|}{|P-Q|} \geq 1-\frac{|P-A|}{|P-Q|} \geq 1-c_{K} \frac{\rho}{n},
$$

since $|P-A|<3 \rho$ and $|P-Q|>n / c_{K}$, by (5.38), (5.41), (5.42).

Next we split $P$ in order to "climb" one step of the staircase (see Fig. 1). Define $x:=$ $\cos \theta_{A}, y:=\sin \theta_{A}$ and

$$
a:=\frac{x}{k}+i \frac{y}{s}
$$


as in (5.15). Moreover set

$$
Q_{1}:=\lambda_{1}\left(a, d_{1}(\bar{a})\right), \quad Q_{2}:=\lambda_{2}\left(-a, d_{2}(\bar{a})\right) .
$$

Here $\lambda_{1}, \lambda_{2}$ are chosen as in (5.10), so that $Q_{j} \in T_{j}$ and, by Lemma 5.4, $\operatorname{det}\left(Q_{j}-J R\right)=0$. Furthermore, set

$$
\left\{\begin{aligned}
\mu_{2} & :=\frac{M_{2}-(t-n) M_{2}}{2 n+M_{2}+(t-n)\left(2-M_{2}\right)}, \\
\mu_{3} & :=\frac{M_{1}-(t-n) M_{1}}{2(n+1)},
\end{aligned}\right.
$$

with $M_{j}$ as in (5.17). With the above choices we have

$$
\left\{\begin{array}{l}
t J R=\mu_{2} t Q_{1}+\left(1-\mu_{2}\right) \tilde{P}, \\
\tilde{P}=\mu_{3}(n+1) Q_{2}+\left(1-\mu_{3}\right)(n+1) J R,
\end{array}\right.
$$

and $\mu_{2}, \mu_{3} \in[0,1]$ by (5.18). In order to check (5.46), we solve the first equation in $\tilde{P}$ to get

$$
\gamma_{2} t J R+\left(1-\gamma_{2}\right) t Q_{1}=\gamma_{3}(n+1) Q_{2}+\left(1-\gamma_{3}\right)(n+1) J R,
$$

with $\mu_{2}=1-1 / \gamma_{2}$ and $\mu_{3}=\gamma_{3}$. Equating the first conformal coordinate of both sides of (5.47) yields

$$
\gamma_{2}=1+\gamma_{3} \frac{n+1}{t} \frac{\lambda_{2}}{\lambda_{1}} .
$$

Substituting (5.48) in the second component of (5.47) gives us

$$
\gamma_{3}\left(\lambda_{1}+\lambda_{2}-\lambda_{1} \lambda_{2}\left(d_{1}(a)+d_{2}(a)\right) r^{-1}\right)=\frac{1-(t-n)}{n+1} \lambda_{1} .
$$

By (5.15), $d_{1}(a)+d_{2}(a)=2 r$ and equation (5.49) yields

$$
\gamma_{3}=\frac{1-(t-n)}{n+1} \frac{\lambda_{1}}{\lambda_{1}+\lambda_{2}-2 \lambda_{1} \lambda_{2}}=\frac{1-(t-n)}{2(n+1)} M_{1} .
$$

Equations (5.48) and (5.50) give us (5.45). Therefore, by (5.40) and (5.46), the measure

$$
v_{A}:=\mu_{1} \delta_{Q}+\left(1-\mu_{1}\right)\left(\mu_{2} \delta_{t} Q_{1}+\left(1-\mu_{2}\right)\left(\mu_{3} \delta_{(n+1)} Q_{2}+\left(1-\mu_{3}\right) \delta_{(n+1) J R}\right)\right)
$$

defines a laminate of third order with barycenter $A$, supported in $T_{1} \cup T_{2} \cup \mathcal{S}_{n+1}$ and such that spt $v_{A} \cap \mathcal{S}_{n+1}=\{(n+1) J R\}$ with $R=R_{\theta_{A}}$. Moreover

$$
\text { spt } v_{A} \subset\left\{\xi \in \mathbb{R}^{2 \times 2}: c_{K}^{-1} n<|\xi|<c_{K} n\right\},
$$

since $c_{K}^{-1} n<|Q|<c_{K} n$ by (5.36), (5.41) and

$$
c_{K}^{-1} n<\left|t Q_{1}\right|,\left|(n+1) Q_{2}\right|<c_{K} n
$$

by (5.43), (5.12). Next we prove (5.37) by estimating

$$
v_{A}\left(\mathcal{S}_{n+1}\right)=\mu_{1}\left(1-\mu_{2}\right)\left(1-\mu_{3}\right) .
$$

Notice that $v_{A}\left(\mathcal{S}_{n+1}\right)$ depends on $R$. For small $\rho$, we have

$$
\mu_{2}=\frac{M_{2}}{2 n}+\rho O\left(\frac{1}{n}\right), \quad \mu_{3}=\frac{M_{1}}{2 n}+\rho O\left(\frac{1}{n}\right),
$$


so that

$$
\left(1-\mu_{2}\right)\left(1-\mu_{3}\right)=1-\frac{M_{1}+M_{2}}{2 n}+\rho O\left(\frac{1}{n^{2}}\right)=1-\frac{1+l}{n}+\rho O\left(\frac{1}{n^{2}}\right),
$$

with $l$ as in (5.17). Although this gives the right asymptotic, we will need to estimate (5.51) for every $n \in \mathbb{N}$. By direct calculation

$$
\left(1-\mu_{2}\right)\left(1-\mu_{3}\right)=\frac{n+(t-n)}{n+1} \frac{2 n+2-M_{1}+(t-n) M_{1}}{2 n+M_{2}+(t-n)\left(2-M_{2}\right)},
$$

so that

$$
\left(1-\mu_{2}\right)\left(1-\mu_{3}\right)=\left(1+\frac{t-n}{n}\right)\left(1-\frac{1}{n+1}\right)\left(1-\frac{2 l(1-(t-n))}{2 n+M_{2}+(t-n)\left(2-M_{2}\right)}\right) .
$$

Let us bound (5.52) from above. Recall that $t-n<\rho<1$ and $2-M_{2}>0$, by (5.18), so the denominator of the third factor in (5.52) is bounded from above by $2(n+1)$ and

$$
\begin{aligned}
\left(1-\mu_{2}\right)\left(1-\mu_{3}\right) & \leq\left(1+\frac{\rho}{n}\right)\left(1-\frac{1}{n+1}\right)\left(1-\frac{l}{n+1}+l \frac{\rho}{n+1}\right) \\
& \leq\left(1+c_{K} \frac{\rho}{n}\right)\left(1-\frac{1}{n+1}\right)\left(1-\frac{l}{n+1}\right)
\end{aligned}
$$

where $c_{K}>1$ is such that

$$
l \frac{\rho}{n+1}\left(1+\frac{\rho}{n}\right) \leq\left(c_{K}-1\right) \frac{\rho}{n}\left(1-\frac{l}{n+1}\right) .
$$

Moreover

$$
\left(1-\frac{1}{n+1}\right)\left(1-\frac{l}{n+1}\right)=1-\frac{1+l}{n+1}+\frac{l}{(n+1)^{2}} \leq 1-\frac{1+l}{n+2}=\beta_{n+2}(R) .
$$

The upper bound in (5.37) follows from (5.53) and (5.54).

Let us now bound (5.52) from below. We can estimate from below the denominator in the third factor of (5.52) with $2 n$, since $t-n>-\rho$ by (5.43) and the assumption that $\rho<m$ with $m$ as in (5.17). Therefore

$$
\begin{aligned}
\left(1-\mu_{2}\right)\left(1-\mu_{3}\right) & \geq\left(1-\frac{\rho}{n}\right)\left(1-\frac{1}{n+1}\right)\left(1-\frac{l}{n}-l \frac{\rho}{n}\right) \\
& \geq\left(1-c_{K} \frac{\rho}{n}\right)\left(1-\frac{1}{n+1}\right)\left(1-\frac{l}{n}\right),
\end{aligned}
$$

if we choose $c_{K}>1$ such that

$$
\left(1-\frac{\rho}{n}\right) l \leq\left(c_{K}-1\right)\left(1-\frac{l}{n}\right) .
$$

Finally

$$
\left(1-\frac{1}{n+1}\right)\left(1-\frac{l}{n}\right) \geq 1-\frac{1+l}{n}=\beta_{n}(R) .
$$

The lower bound in (5.37) follows from (5.55) and (5.56). 
Finally, the last part of the statement follows from a simple geometrical argument, recalling that $\arg R=\theta_{A}=-\arg \left(b-d_{1}(\bar{a})\right)$ and using hypothesis (5.38).

Remark 5.7 By iteratively applying Lemma 5.6, one can obtain, for every $R_{\theta} \in S O(2)$, a sequence of laminates of finite order $v_{n} \in \mathcal{L}\left(\mathbb{R}^{2 \times 2}\right)$ that satisfies $\bar{v}_{n}=J R_{\theta}$, spt $v_{n} \subset$ $T_{1} \cup T_{2} \cup \mathcal{S}_{n+1}$, and

$$
\lim _{n \rightarrow \infty} \int_{\mathbb{R}^{2 \times 2}}|\lambda|^{p\left(R_{\theta}\right)} d v_{n}(\lambda)=\infty,
$$

where $p\left(R_{\theta}\right) \in\left[\frac{2 S}{S+1}, \frac{2 K}{K+1}\right]$ is the function defined in (5.17). Indeed, setting $A=J R_{\theta}$ and iterating the construction of Lemma 5.6, yields $v_{n} \in \mathcal{L}\left(\mathbb{R}^{2 \times 2}\right)$ such that $\bar{v}_{n}=J R_{\theta}$ and spt $v_{n} \subset T_{1} \cup T_{2} \cup \mathcal{S}_{n+1}$. Notice that $v_{n}$ contains the term $\prod_{j=1}^{n}\left(1-\mu_{2}^{j}\right)\left(1-\mu_{3}^{j}\right) \delta_{(n+1) J R_{\theta}}$, with $\mu_{2}^{j}, \mu_{3}^{j}$ as defined in (5.45). Therefore, using (5.19) and (5.37) (with $\rho=0$ ), we obtain

$$
\prod_{j=1}^{n}\left(1-\mu_{2}^{j}\right)\left(1-\mu_{3}^{j}\right) \approx \prod_{j=1}^{n} \beta_{j}\left(R_{\theta}\right) \approx \frac{1}{n^{p\left(R_{\theta}\right)}}
$$

which implies (5.57).

Remark 5.8 In the isotropic case $S=K$, the laminate $v_{A}$ provided by Lemma 5.6 coincides with the one in [2, Lemma 3.16]. In particular, the growth condition (5.37) is independent of the initial point $A$, and it reads as

$$
\left(1-c_{K} \frac{\rho}{n}\right) \beta_{n}(I) \leq v_{A}\left(\mathcal{S}_{n+1}\right) \leq\left(1+c_{K} \frac{\rho}{n}\right) \beta_{n+2}(I), \quad \beta_{n}(I)=1-\frac{1+k}{n} .
$$

Moreover, by Remark 5.7, for every $R_{\theta} \in S O(2), J R_{\theta}$ is the center of mass of a sequence of laminates of finite order such that (5.57) holds with $p\left(R_{\theta}\right) \equiv \frac{2 K}{K+1}$, which gives the desired growth rate.

In contrast, in the anisotropic case $1<S<K$, the growth rate of the laminates explicitly depends on the argument of the barycenter $J R_{\theta}$. The desired growth rate corresponds to $\theta=0$, that is, the center of mass has to be $J$.

In constructing approximate solutions with the desired integrability properties, it is then crucial to be able to select rotations whose angle lies in an arbitrarily small neighbourhood of $\theta=0$.

We now proceed to show the existence of a piecewise affine map $f$ that solves the differential inclusion (4.2) up to an arbitrarily small $L^{\infty}$ error. Such map will have the integrability properties given by (5.59).

Proposition 5.9 Let $\Omega \subset \mathbb{R}^{2}$ be an open bounded domain. Let $K>1, \alpha \in(0,1), \varepsilon>0$, $0<\delta_{0}<\frac{2 K}{K+1}-\frac{2 S}{S+1}, \gamma>0$. There exist a constant $c_{K, \delta_{0}}>1$, depending only on $K, S_{1}, S_{2}, \delta_{0}$, and a piecewise affine map $f \in W^{1,1}\left(\Omega ; \mathbb{R}^{2}\right) \cap C^{\alpha}\left(\bar{\Omega} ; \mathbb{R}^{2}\right)$, such that

(i) $f(x)=J x$ on $\partial \Omega$,

(ii) $[f-J x]_{C^{\alpha}(\bar{\Omega})}<\varepsilon$,

(iii) $\operatorname{dist}(\nabla f(x), T)<\gamma$ a.e. in $\Omega$.

Moreover

$$
\frac{1}{c_{K, \delta_{0}}} t^{-\frac{2 K}{K+1}}<\frac{|\{x \in \Omega:|\nabla f(x)|>t\}|}{|\Omega|}<c_{K, \delta_{0}} t^{-p},
$$


where $p \in\left(\frac{2 K}{K+1}-\delta_{0}, \frac{2 K}{K+1}\right]$. That is, $\nabla f \in L_{\text {weak }}^{p}\left(\Omega ; \mathbb{R}^{2 \times 2}\right)$ and $\nabla f \notin L^{\frac{2 K}{K+1}}\left(\Omega ; \mathbb{R}^{2 \times 2}\right)$. In particular $f \in W^{1, q}\left(\Omega ; \mathbb{R}^{2}\right)$ for every $q<$ p but $\int_{\Omega}|\nabla f(x)|^{\frac{2 K}{K+1}} d x=\infty$.

Proof By Lemma 5.5 the function $p:(-\pi, \pi] \rightarrow\left[\frac{2 S}{S+1}, \frac{2 K}{K+1}\right]$ is uniformly continuous. Let $\alpha:[0, \infty] \rightarrow[0, \infty]$ be its modulus of continuity. Fix $0<\delta<\pi / 4$ such that

$$
\alpha(\delta)<\delta_{0} .
$$

Let $\left\{\rho_{n}\right\}$ be a strictly decreasing positive sequence satisfying

$$
\rho_{1}<\frac{1}{4} \min \left\{m, c_{K}^{-1}, \operatorname{dist}\left(\mathcal{S}_{1}, T\right), \gamma\right\}, \quad \rho_{n}<\frac{\delta}{4} 2^{-n},
$$

where $m>0$ and $c_{K}>1$ are the constants from Lemma 5.6. Define $\left\{\delta_{n}\right\}$ as

$$
\delta_{1}:=0 \quad \text { and } \quad \delta_{n}:=\sum_{j=1}^{n-1} \rho_{n} \text { for } n \geq 2 .
$$

In particular from (5.61),(5.62) it follows that

$$
\delta_{n}<\frac{\delta}{2}, \quad \text { for every } n \in \mathbb{N} \text {. }
$$

Step 1. Similarly to the proof of [2, Proposition 3.17], by repeatedly combining Lemma 5.6 and Proposition 3.2, we will prove the following statement:

Claim. There exist sequences of piecewise constant functions $\tau_{n}: \Omega \rightarrow(0, \infty)$ and piecewise affine Lipschitz mappings $f_{n}: \Omega \rightarrow \mathbb{R}^{2}$, such that

(a) $f_{n}(x)=J x$ on $\partial \Omega$,

(b) $\left[f_{n}-J x\right]_{C^{\alpha}(\bar{\Omega})}<\left(1-2^{-n}\right) \varepsilon$,

(c) $\operatorname{dist}\left(\nabla f_{n}(x), T \cup \mathcal{S}_{n}^{\delta_{n}}\right)<\tau_{n}(x)$ a.e. in $\Omega$,

(d) $\tau_{n}(x)=\rho_{n}$ in $\Omega_{n}$,

where

$$
\Omega_{n}:=\left\{x \in \Omega: \operatorname{dist}\left(\nabla f_{n}(x), T\right) \geq \rho_{n}\right\}
$$

Moreover

$$
\prod_{j=1}^{n-1}\left(1-c_{K} \frac{\rho_{j}}{j}\right) \beta_{j}\left(R_{0}\right) \leq \frac{\left|\Omega_{n}\right|}{|\Omega|} \leq \prod_{j=1}^{n-1}\left(1+c_{K} \frac{\rho_{j}}{j}\right) \beta_{j+2}\left(R_{\delta}\right) .
$$

Proof of the claim. We proceed by induction. Set $f_{1}(x):=J x$ and $\tau_{1}(x):=\rho_{1}$ for every $x \in \Omega$. Since $J \in \mathcal{S}_{1}^{0}$, then $f_{1}$ satisfies (a)-(c). Also, $\rho_{1}<\operatorname{dist}\left(T, \mathcal{S}_{1}\right) / 4$ by (5.61), so $\Omega_{1}=\Omega$ and (d), (5.64) follow.

Assume now that $f_{n}$ and $\tau_{n}$ satisfy the inductive hypothesis. We will first define $f_{n+1}$ by modifying $f_{n}$ on the set $\Omega_{n}$. Since $f_{n}$ is piecewise affine we have a decomposition of $\Omega_{n}$ into pairwise disjoint open subsets $\Omega_{n, i}$ such that

$$
\left|\Omega_{n} \backslash \bigcup_{i=1}^{\infty} \Omega_{n, i}\right|=0
$$

with $f_{n}(x)=A_{i} x+b_{i}$ in $\Omega_{n, i}$, for some $A_{i} \in \mathbb{R}^{2 \times 2}$ and $b_{i} \in \mathbb{R}^{2}$. Moreover

$$
\operatorname{dist}\left(A_{i}, \mathcal{S}_{n}^{\delta_{n}}\right)<\rho_{n}
$$


by (c) and (d). Since (5.66) and (5.61) hold, we can invoke Lemma 5.6 to obtain a laminate $v_{A_{i}}$ and a rotation $R^{i}=R_{\theta_{A_{i}}}$ satisfying, in particular, $\bar{v}_{A_{i}}=A_{i}$,

$$
\begin{aligned}
& \left|\arg R^{i}\right|=\left|\theta_{A_{i}}\right|<\delta_{n+1}, \\
& \text { spt } v_{A_{i}} \subset T \cup \mathcal{S}_{n+1}^{\delta_{n+1}},
\end{aligned}
$$

since $\delta_{n+1}=\delta_{n}+\rho_{n}$ by (5.62). By applying Proposition 3.2 to $v_{A_{i}}$ and by taking into account (5.68), we obtain a piecewise affine Lipschitz mapping $g_{i}: \Omega_{n, i} \rightarrow \mathbb{R}^{2}$, such that

(e) $g_{i}(x)=A_{i} x+b_{i}$ on $\partial \Omega_{n, i}$,

(f) $\left[g_{i}-f_{n}\right]_{C^{\alpha}\left(\overline{\Omega_{n, i}}\right)}<2^{-(n+1+i)} \varepsilon$,

(g) $c_{K}^{-1} n<\left|\nabla g_{i}(x)\right|<c_{K} n$ a.e. in $\Omega_{n, i}$,

(h) $\operatorname{dist}\left(\nabla g_{i}(x), T \cup \mathcal{S}_{n+1}^{\delta_{n+1}}\right)<\rho_{n+1}$ a.e. in $\Omega_{n, i}$.

Moreover

$$
\left(1-c_{K} \frac{\rho_{n}}{n}\right) \beta_{n}\left(R^{i}\right) \leq \frac{\left|\omega_{n, i}\right|}{\left|\Omega_{n, i}\right|} \leq\left(1+c_{K} \frac{\rho_{n}}{n}\right) \beta_{n+2}\left(R^{i}\right),
$$

with

$$
\omega_{n, i}:=\left|\left\{x \in \Omega_{n, i}: \operatorname{dist}\left(\nabla g_{i}(x), \mathcal{S}_{n+1}^{\delta_{n+1}}\right)<\rho_{n+1}\right\}\right| .
$$

Set

$$
f_{n+1}(x):= \begin{cases}f_{n}(x) & \text { if } x \in \Omega \backslash \Omega_{n}, \\ g_{i}(x) & \text { if } x \in \Omega_{n, i} .\end{cases}
$$

Since $\Omega_{n+1}$ is well defined, we can also introduce

$$
\tau_{n+1}(x):= \begin{cases}\tau_{n}(x) & \text { for } x \in \Omega \backslash \Omega_{n+1}, \\ \rho_{n+1} & \text { for } x \in \Omega_{n+1},\end{cases}
$$

so that (d) holds. From (e) we have $f_{n+1}(x)=J x$ on $\partial \Omega$. From (f) we get $\left[f_{n+1}-f_{n}\right]_{C^{\alpha}(\bar{\Omega})}<$ $2^{-(n+1)} \varepsilon$ so that (b) follows. (c) is a direct consequence of (d), (h), and the fact that $\rho_{n}$ is strictly decreasing. Finally let us prove (5.64). First notice that the sets $\omega_{n, i}$ are pairwise disjoint. By (5.61), in particular we have $\rho_{n+1}<\operatorname{dist}\left(T, \mathcal{S}_{1}\right) / 4$, so that

$$
\left|\Omega_{n+1} \backslash \bigcup_{i=1}^{\infty} \omega_{n, i}\right|=0 \text {. }
$$

By (5.67) and (5.63) we have $\left|\arg R^{i}\right|<\delta$. Then by the properties of $\beta_{n}$ (see Lemma 5.5),

$$
\beta_{n}\left(R^{i}\right) \geq \beta_{n}\left(R_{0}\right) \text { and } \beta_{n+2}\left(R^{i}\right) \leq \beta_{n+2}\left(R_{\delta}\right) .
$$

Using (5.71), (5.65), (5.70) in (5.64) yields

$$
\left|\Omega_{n}\right|\left(1-c_{K} \frac{\rho_{n}}{n}\right) \beta_{j}\left(R_{0}\right) \leq\left|\Omega_{n+1}\right| \leq\left|\Omega_{n}\right|\left(1+c_{K} \frac{\rho_{n}}{n}\right) \beta_{j+2}\left(R_{\delta}\right),
$$

and (5.64) follows.

Step 2. Notice that on $\Omega \backslash \Omega_{n}$ we have that $\nabla f_{n+1}=\nabla f_{n}$ almost everywhere, so $\Omega_{n+1} \subset \Omega_{n}$. Therefore $\left\{f_{n}\right\}$ is obtained by modification on a nested sequence of open sets, satisfying

$$
\prod_{j=1}^{n-1}\left(1-c_{K} \frac{\rho_{j}}{j}\right) \beta_{j}\left(R_{0}\right) \leq \frac{\left|\Omega_{n}\right|}{|\Omega|} \leq \prod_{j=1}^{n-1}\left(1+c_{K} \frac{\rho_{j}}{j}\right) \beta_{j+2}\left(R_{\delta}\right) .
$$


By (5.61) we have $\rho_{n}<\min \left\{2^{-n} \delta, c_{K}^{-1}\right\} / 4$, so that

$$
\prod_{j=1}^{\infty}\left(1-c_{K} \frac{\rho_{j}}{j}\right)=c_{1}, \quad \prod_{j=1}^{\infty}\left(1+c_{K} \frac{\rho_{j}}{j}\right)=c_{2},
$$

with $0<c_{1}<c_{2}<\infty$, depending only on $K, S_{1}, S_{2}, \delta$ (and hence from $\delta_{0}$, by (5.60)). Moreover, from Lemma 5.5,

$$
\prod_{j=1}^{n} \beta_{j}\left(R_{\theta}\right)=n^{-p\left(R_{\theta}\right)}+O\left(\frac{1}{n}\right), \quad \text { uniformly in }(-\pi, \pi] .
$$

Therefore, there exists a constant $c_{K, \delta_{0}}>1$ depending only on $K, S_{1}, S_{2}, \delta_{0}$, such that

$$
\frac{1}{c_{K, \delta_{0}}} n^{-\frac{2 K}{K+1}} \leq\left|\Omega_{n}\right| \leq c_{K, \delta_{0}} n^{-p_{\delta_{0}}}
$$

since $p\left(R_{0}\right)=\frac{2 K}{K+1}$. Here $p_{\delta_{0}}:=p\left(R_{\delta}\right)$. Notice that, by (5.60), $p_{\delta_{0}} \in\left(\frac{2 K}{K+1}-\delta_{0}, \frac{2 K}{K+1}\right]$, since $p$ is strictly decreasing in $[0, \pi / 2]$.

From (5.72), in particular we deduce $\left|\Omega_{n}\right| \rightarrow 0$. Therefore $f_{n} \rightarrow f$ almost everywhere in $\Omega$, with $f$ piecewise affine. Furthermore $f$ satisfies (i)-(iii) by construction.

We are left to estimate the distribution function of $\nabla f$. By $(\mathrm{g})$ we have that

$$
|\nabla f(x)|>\frac{n}{c_{K, \delta_{0}}} \quad \text { in } \quad \Omega_{n} \quad \text { and } \quad|\nabla f(x)|<c_{K, \delta_{0}} n \text { in } \Omega \backslash \Omega_{n} .
$$

For a fixed $t>c_{K, \delta_{0}}$, let $n_{1}:=\left[c_{K, \delta_{0}} t\right]$ and $n_{2}:=\left[c_{K, \delta_{0}}^{-1} t\right]$, where $[\cdot]$ denotes the integer part function. Therefore

$$
\Omega_{n_{1}+1} \subset\{x \in \Omega:|\nabla f(x)|>t\} \subset \Omega_{n_{2}}
$$

and (5.59) follows from (5.72), with $p=p_{\delta_{0}}$. Lastly, (5.59) implies that $\nabla f_{n}$ is uniformly bounded in $L^{1}$, so that $f \in W^{1,1}\left(\Omega ; \mathbb{R}^{2}\right)$ by dominated convergence.

We remark that the constant $c_{K, \delta_{0}}$ in (5.59) is monotonically increasing as a function of $\delta_{0}$, that is $c_{K, \delta_{1}} \leq c_{K, \delta_{2}}$ if $\delta_{1} \leq \delta_{2}$.

We now proceed with the construction of exact solutions to (4.2). We will follow a standard argument (see, e.g., [5, Remark 6.3], [9, Thoerem A.2]).

Theorem 5.10 Let $\sigma_{1}, \sigma_{2}$ be defined by (1.2) for some $K, S_{1}, S_{2}$ as in (5.3) and $S$ as in (4.7). There exist coefficients $\sigma_{n} \in L^{\infty}\left(\Omega ;\left\{\sigma_{1}, \sigma_{2}\right\}\right)$, exponents $p_{n} \in\left[\frac{2 S}{S+1}, \frac{2 K}{K+1}\right]$, functions $u_{n} \in W^{1,1}(\Omega ; \mathbb{R})$, such that

$$
\begin{aligned}
& \begin{cases}\operatorname{div}\left(\sigma_{n}(x) \nabla u_{n}(x)\right)=0 & \text { in } \Omega, \\
u_{n}(x)=x_{1} & \text { on } \quad \partial \Omega,\end{cases} \\
& \nabla u_{n} \in L_{\text {weak }}^{p_{n}}\left(\Omega ; \mathbb{R}^{2}\right), \quad p_{n} \rightarrow \frac{2 K}{K+1}, \\
& \nabla u_{n} \notin L^{\frac{2 K}{K+1}}\left(\Omega ; \mathbb{R}^{2}\right) .
\end{aligned}
$$

In particular $u_{n} \in W^{1, q}(\Omega ; \mathbb{R})$ for every $q<p_{n}$, but $\int_{\Omega}\left|\nabla u_{n}\right|^{\frac{2 K}{K+1}} d x=\infty$. 
Proof By Proposition 5.9 there exist sequences $f_{n} \in W^{1,1}\left(\Omega ; \mathbb{R}^{2}\right) \cap C^{\alpha}\left(\bar{\Omega} ; \mathbb{R}^{2}\right), \gamma_{n} \searrow 0$, $p_{n} \in\left[\frac{2 S}{S+1}, \frac{2 K}{K+1}\right]$, such that, $f_{n}(x)=J x$ on $\partial \Omega$,

$$
\begin{aligned}
& \operatorname{dist}\left(\nabla f_{n}(x), T_{1} \cup T_{2}\right)<\gamma_{n} \text { a.e. in } \Omega, \\
& \nabla f_{n} \in L_{\text {weak }}^{p_{n}}\left(\Omega ; \mathbb{R}^{2 \times 2}\right), \quad p_{n} \rightarrow \frac{2 K}{K+1}, \quad \nabla f_{n} \notin L^{\frac{2 K}{K+1}}\left(\Omega ; \mathbb{R}^{2 \times 2}\right) .
\end{aligned}
$$

In euclidean coordinates, condition (5.76) implies that

$$
\left(\begin{array}{c}
\nabla f_{n}^{1}(x) \\
\nabla f_{n}^{2}(x)
\end{array}\right)=\left(\begin{array}{c}
E_{n}(x) \\
R_{\frac{\pi}{2}} \sigma_{n}(x) E_{n}(x)
\end{array}\right)+\left(\begin{array}{l}
a_{n}(x) \\
b_{n}(x)
\end{array}\right) \text { a.e. in } \Omega
$$

with $f_{n}=\left(f_{n}^{1}, f_{n}^{2}\right), \sigma_{n}:=\sigma_{1} \chi_{\left\{\nabla f \in T_{1}\right\}}+\sigma_{2} \chi_{\left\{\nabla f \in T_{2}\right\}}, E_{n}: \Omega \rightarrow \mathbb{R}^{2}, R_{\frac{\pi}{2}}=\left(\begin{array}{cc}0 & -1 \\ 1 & 0\end{array}\right)$ and

$$
a_{n}, b_{n} \rightarrow 0 \quad \text { in } \quad L^{\infty}\left(\Omega ; \mathbb{R}^{2}\right) .
$$

The boundary condition $f_{n}=J x$ reads $f_{n}^{1}=x_{1}$ and $f_{n}^{2}=-x_{2}$. We set $u_{n}:=f_{n}^{1}+v_{n}$, where $v_{n} \in H_{0}^{1}(\Omega, \mathbb{R})$ is the unique solution to

$$
\operatorname{div}\left(\sigma_{n} \nabla v\right)=-\operatorname{div}\left(\sigma_{n} a_{n}-R_{\frac{\pi}{2}}^{T} b_{n}\right) .
$$

Notice that $v_{n}$ is uniformly bounded in $H^{1}$ by (5.79). Since (5.78) holds, it is immediate to check that $\operatorname{div}\left(\sigma_{n} \nabla u_{n}\right)=\operatorname{div}\left(R_{\frac{\pi}{2}}^{T} \nabla f_{n}^{2}\right)=0$, so that $u_{n}$ is a solution of (5.73). Finally, the regularity thesis (5.74), (5.75), follows from the definition of $u_{n}$ and the fact that $v_{n} \in$ $H_{0}^{1}(\Omega ; \mathbb{R})$ and $f_{n}^{1}$ satisfies $(5.77)$ with $1<p_{n}<2$.

Open Access This article is distributed under the terms of the Creative Commons Attribution 4.0 International License (http://creativecommons.org/licenses/by/4.0/), which permits unrestricted use, distribution, and reproduction in any medium, provided you give appropriate credit to the original author(s) and the source, provide a link to the Creative Commons license, and indicate if changes were made.

\section{References}

1. Astala, K.: Area distortion of quasiconformal mappings. Acta Math. 173(1), 37-60 (1994)

2. Astala, K., Faraco, D., Székelyhidi, L.: Convex integration and the $L^{p}$ theory of elliptic equations. Ann. Scuola Norm. Sup. Pisa Cl. Sci. 5(7), 1-50 (2008)

3. Conti, S., Faraco, D., Maggi, F.: A new approach to counterexamples to $L^{1}$ estimates: Korn's inequality, geometric rigidity, and regularity for gradients of separately convex functions. Arch. Ration. Mech. Anal. 175(2), 287-300 (2005)

4. Conti, S., Faraco, D., Maggi, F., Müller, S.: Rank-one convex functions on $2 \times 2$ symmetric matrices and laminates on rank-three lines. Calc. Var. Partial Differ. Equ. 24(4), 479-493 (2005)

5. Faraco, D.: Milton's conjecture on the regularity of solutions to isotropic equations. Ann. Inst. H. Poincaré Anal. Non Linéaire 20(5), 889-909 (2003)

6. Faraco, D., Mora-Corral, C., Oliva, M.: Sobolev homeomorphisms with gradients of low rank via laminates. Adv. Calc. Var. (2016). doi:10.1515/acv-2016-0009

7. Müller, S.: Variational models for microstructure and phase transitions. In: Calculus of Variations and Geometric Evolution Problems (Cetraro, 1996), pp. 85-210 (1999)

8. Müller, S., Šverák, V.: Convex integration for Lipschitz mappings and counterexamples to regularity. Ann. Math. 157(3), 715-742 (2003)

9. Nesi, V., Palombaro, M., Ponsiglione, M.: Gradient integrability and rigidity results for two-phase conductivities in two dimensions. Ann. Inst. H. Poincaré Anal. Non Linéaire 31(3), 615-638 (2014) 
10. Oliva, M.: Bi-Sobolev homeomorphisms $f$ with $d f$ and $d f^{-1}$ of low rank using laminates. Calc. Var. Partial Differ. Equ. 55(6), 55-135 (2016)

11. Pedregal, P.: Laminates and microstructure. Eur. J. Appl. Math. 4(2), 121-149 (1993)

12. Petermichl, S., Volberg, A.: Heating of the Ahlfors-Beurling operator: weakly quasiregular maps on the plane are quasiregular. Duke Math. J. 112, 281-305 (2002) 